\title{
The IRI topside parameters
}

\section{P. Coïsson and S. M. Radicella}

Abdus Salam ICTP, strada costiera 11, 34014 Trieste, Italy

\begin{abstract}
The IRI electron density topside has been constructed on the basis of the data available about thirty years ago. Recently a large amount of data from old topside sounders have been processed to get electron density profiles. These profiles allow to test IRI model under a wide spectrum of different conditions and to understand the behavior of the modeled topside. A set of 12000 topside profiles have been chosen from the ISIS2 database, selecting those with the better quality of the inverted profile. An analysis of each IRI topside constitutive parameter has been done for all the experimental cases allowing to find some critical conditions under which the IRI topside could become completely unrealistic. In particular for very high solar activity at high latitudes it has been found that some IRI parameters could reach values that change drastically the shape of IRI topside. A proposal to control their range of variability is formulated in order to avoid the occurrence of such cases.
\end{abstract}

\section{Introduction}

The IRI topside has been constructed following the Booker's approach (Bilitza, 1990), on the basis of the data available at that time. Since its original formulation, IRI model has been constantly checked against all new experimental data sources available (Bilitza, 2001). Recently a big effort to analyze large amounts of old satellite topside soundings has made available to the scientific community new databases of experimental topside electron density profiles (Huang et al., 2002; Bilitza et al., 2002).

In a previous work by the same authors (Coïsson et al., 2002) IRI electron density has been tested against experimental profiles. The IRI topside profile has been compute for all the experimental conditions in which the topside soundings were recorded. For high solar activity at high latitude, cases have been found in which IRI topside electron den-

Correspondence to: P. Coïsson

coissonp@ictp.trieste.it sity is constant at all heights. The present work analyzes the causes of this particular IRI feature, focusing the attention on each parameter concurring in the characterization of IRI topside.

\section{The IRI topside formulation}

The IRI topside formulation is based on the approach to model the profile using different sections with nearly constant gradients. The analytical representation (Bilitza, 1990) is an exponential layer modulated by the Booker function $B(x ; x, M, T G, T X, T C)$ :

$N(h)=N m F 2 \exp \left(-\frac{1}{\alpha} B\left(x ; x_{0}, 2, T G, T X, T C\right)\right)$

where:

$B\left(x ; x_{0}, M, T G, T X, T C\right)=$

$\left(x-x_{0}\right) T G_{1}+\sum_{j=1}^{M}\left(T G_{j+1}-T G_{j}\right) T C_{j}$.

$\ln \left[\left(1+\exp \left(\frac{x-T X_{j}}{T C_{j}}\right)\right) /\left(1+\exp \left(\frac{x_{0}-T X_{j}}{T C_{j}}\right)\right)\right]$

$x$ is a modified altitude variable transforming the F2-peak to near $300 \mathrm{~km}$ :

$x-x_{0}=\alpha(h-h m F 2)$

with the transformation factor $\alpha$ :

$\alpha=\frac{700}{1000-h m F 2}$

and the new peak height $x_{0}$ :

$x_{0}=300-\delta$

The parameters appearing in the Booker function: gradients $T G$, sections boundaries $T X$ and thicknesses of transition zone between sections $T C$ are recorded in Table 1. Empirical relations based on geomagnetic latitude, $\Phi$, F2 layer critical 


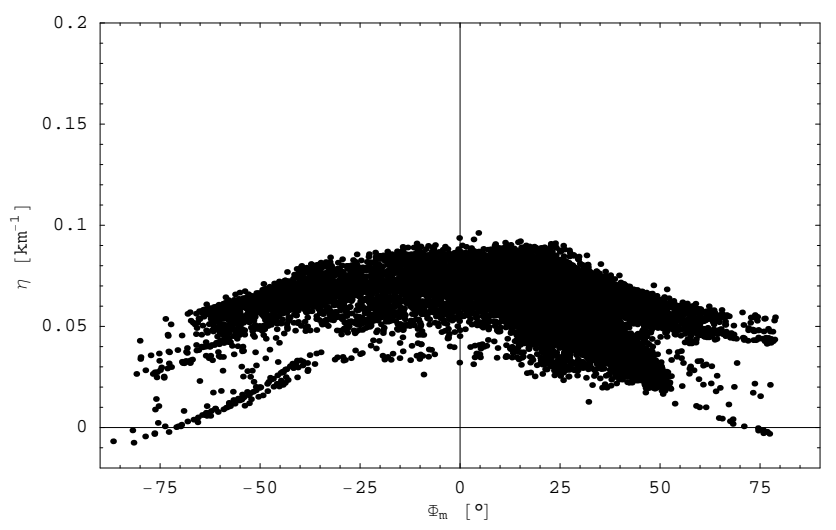

Fig. 1. $\eta$ parameter as function of geomagnetic latitude. Negative values were found for $F 10.7>185$.

Table 1. Parameters used in the IRI topside formulation.

\begin{tabular}{cccc}
\hline$J$ & $T G$ & $T X$ & $T C$ \\
\hline 1 & $-\xi$ & 300 & 100 \\
2 & 0 & 394.5 & $\beta$ \\
3 & $\eta$ & - & - \\
\hline
\end{tabular}

frequency $f o F 2$, and solar flux $F 10.7$ govern their behaviors:

$$
\begin{aligned}
\beta, \eta, \xi= & t_{0}+t_{1} T_{m}(\Phi)+t_{2} R(F 10.7)+t_{3} T(\Phi) R(F 10.7) \\
& +t_{4} \text { foF } 2+t_{5} \text { foF } 2 T(\Phi)+t_{6} \text { foF } 2
\end{aligned}
$$

where

$$
\begin{aligned}
& T(\Phi)=\cos ^{2} \Phi \\
& T_{m}(\Phi)=\left\{\begin{array}{cc}
\frac{\exp (\Phi / 15)}{(1+\exp (\Phi / 15))^{2}} & \text { for } \eta \\
\cos ^{2} \Phi & \text { for } \beta, \xi
\end{array}\right. \\
& R(F 10.7)=\left\{\begin{array}{cc}
(F 10.7-40) / 30 & \text { for } F 10.7<193 \\
5.1 & \text { otherwise }
\end{array}\right.
\end{aligned}
$$

The coefficients $t_{i}$ were determined by fitting these functions to the values obtained from Bent's computations (Bilitza, 1990).

The corrective term $\delta$ is related to the other parameters by:

$\delta=\left(\frac{\eta}{1+Z}-\frac{\xi}{2}\right) /\left(\frac{\eta}{\beta} \frac{Z}{(1+Z)^{2}}+\frac{\xi}{400}\right)$

with

$Z=\exp \left(\frac{94.5}{\beta}\right)$

The values of the parameters $\beta, \eta, \xi$ and $\delta$ were analyzed in this work.

\section{Experimental data}

The database of electron density profiles from the ISIS2 satellite were used for model comparison (Bilitza et al.,

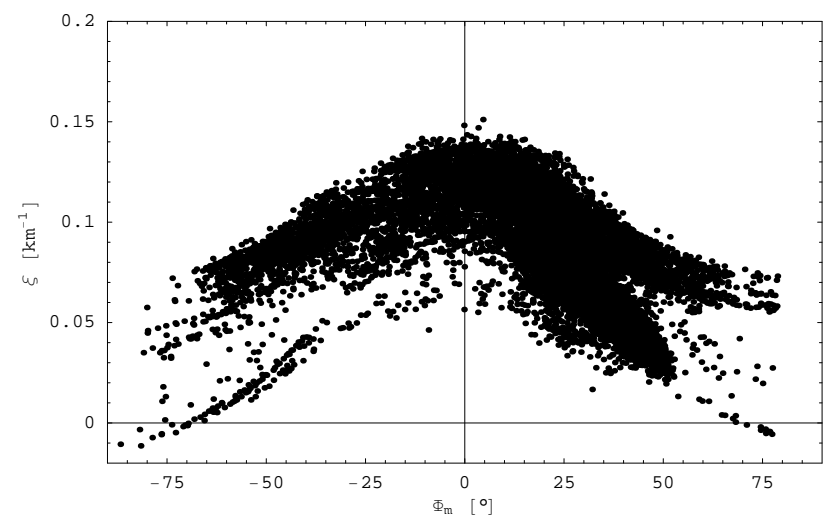

Fig. 2. $\xi$ parameter as function of geomagnetic latitude. Negative values were found for $F 10.7>185$.

2003). 12280 profiles have been selected on the basis of the better profile quality.

IRI was used to compute modeled topside profiles corresponding to the experimental ones: the experimental peak values ( $f o F 2$ and $\mathrm{hmF} 2$ ) were provided as input to the model, in order to focus the comparison on the topside profile shape.

Values of $\eta, \beta, \xi$ and $\delta$ have been extracted to analyze their behaviors as the experimental conditions were changing.

\section{Results}

The available experimental data allowed to find critical conditions for IRI. The attention was focused on high solar activity conditions at high latitudes, since it has been found that under these conditions the experimental profiles were always overestimated and in some cases the IRI electron density was constant at any height. The parameters have been considered to find which ones were responsible of such behavior. It has been found that $\eta$ (Fig. 1) and $\xi$ (Fig. 2), which are supposed to have positive values, could become negative under the above mentioned conditions. The behavior of $\delta$ (Fig. 3) under similar conditions was also very delicate: it could reach very high absolute values and change sign passing through a zero of the denominators of Eq. (10).

It appears that all these conditions concur to change substantially the shape of the topside profile and they should be avoided. As first trial of a topside formulation improvement it has been put a limitation of the values each parameter could reach. It has been found that this values have to be chosen very carefully, since small variations lead to strong changes in the profile shape. A first proposal of limitation may be:

$-\eta \geq 0.02$.

$-\xi \geq 0.015$.

$-\delta$ constructed in such way to avoid the region where it increases (one possibility is to use a limited $\eta$ to compute it). 


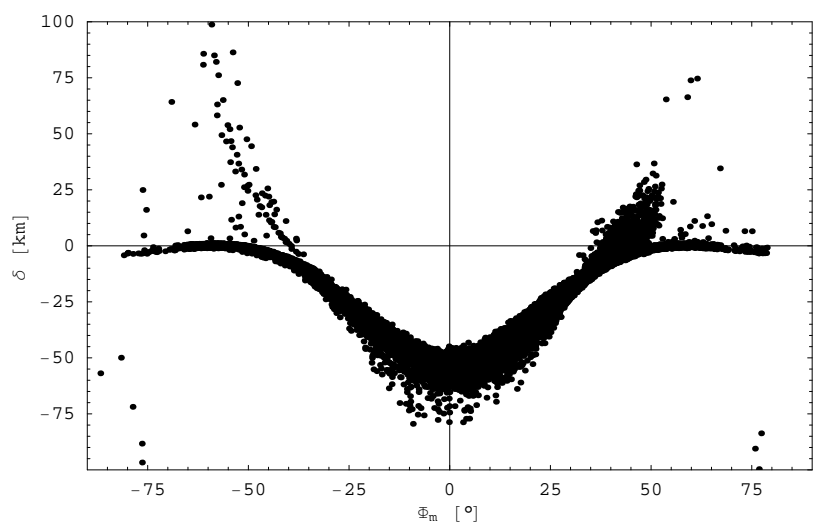

Fig. 3. $\delta$ parameter as function of geomagnetic latitude. Large absolute values were found for $F 10.7>180$.

A test has been performed, showing that this kind of limitations are affecting only profiles at high geomagnetic latitudes for high solar activity. In Fig. 4 is shown an example of experimental profile for conditions in which the IRI profile is nearly constant in the topside. The profiles obtained putting lower limits to $\eta$ and $\xi$ are also shown.

\section{Conclusions}

The parameters governing IRI topside shape have been analyzed for the experimental conditions of ISIS2 soundings and it has been found that during high solar activity at high geomagnetic latitudes some of them could reach values which produce unrealistic profiles. As first solution it is proposed to keep the values of $\xi$ and $\eta$ higher than a minimum value and to avoid $\delta$ reaching too high absolute values. It has been shown that small variations of these minimum values could lead to completely different profiles. Further investigations have to be performed to find the optimum limiting value for each of them.

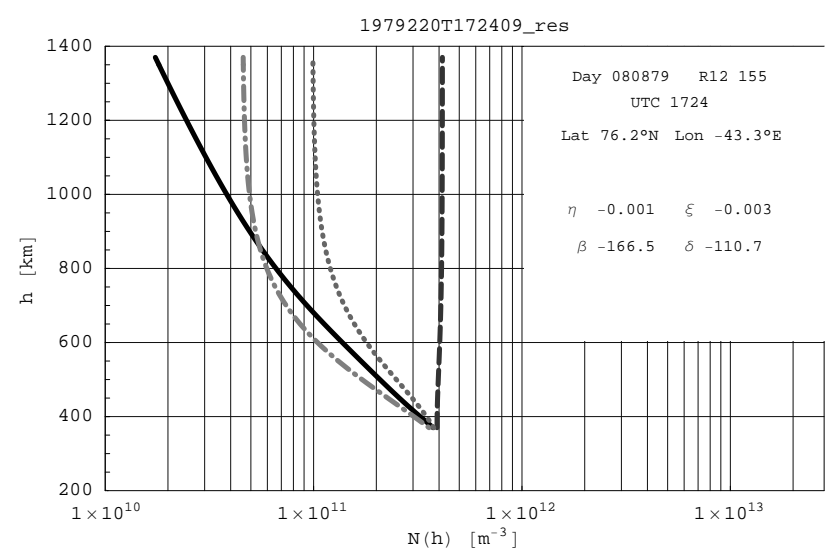

Fig. 4. Example of IRI profile nearly constant at every height, and the profiles obtained limiting the parameters. Solid: experimental profile; Dashed: IRI profile; Dotted: IRI profile limiting $\xi \geq 0.15$ and $\eta \geq 0.15$; Dash-dotted: IRI profile limiting $\xi \geq 0.15$ and $\eta \geq 0.2$.

\section{References}

Bilitza, D.: International Reference Ionosphere 1990, NSSDC 9022, Greenbelt, Maryland, 1990.

Bilitza, D.: International Reference Ionosphere 2000, Radio Science, 36, 2, 261-275, 2001.

Bilitza, D., Huang, X., Reinish, B. W., and Benson, R. F.: TOPIST - Automated Processing of ISIS Topside Ionograms, 2002 Ionospheric Effects Symposium edited by Goodman, J. M., 333-340, 2002.

Bilitza, D., Reinish, B. W., Benson, R. F., Grebowsky, J., Papistashvili, N., Huang, X., Schar, W., and Hills, K.: Online database of satellite sounder and insitu measurements covering two solar cycles, Adv. Space Res., 31, 3, 769-774, 2003.

Coïsson P., Radicella, S. M., and Nava, B.: Comparisons of experimental topside electron concentration profiles with IRI and NeQuick models, Annals of Geophysics, 45, 1, 111-116, 2002.

Huang, X., Reinisch, B. W., Bilitza, D., and Benson, R. F.: Electron density profiles of the topside ionosphere, Annals of Geophysics, 45, 1, 125-130, 2002. 